\title{
Clues to prognosis in multiple sclerosis found in the blood
}

a simple blood
test very early
on in the
course of MS
can predict
the future
of a patient
with MS

Neurofilament light (NfL) and microRNAs (miRNAs) can help to predict the course that multiple sclerosis (MS) will take for individuals, according to two new studies published in Neurology and Acta Neurologica Scandinavica. The markers could help to identify progressive disease and facilitate treatment decisions.

Most current MS treatments are used to minimize inflammatory activity in the relapsing-remitting form of the disease, yet it is disease progression in relapsing-remitting and progressive MS that ultimately leads to accumulation of disability. Reliable markers that identify patients with or at risk of progressive disease would, therefore, be valuable in clinical practice. In the new studies, two potential markers in the blood were investigated.

In the first study, Ali Manouchehrinia and colleagues investigated the association of blood levels of NfL at the time of MS diagnosis with later disability progression. "Several nationwide cohorts of patients and population matched controls have been
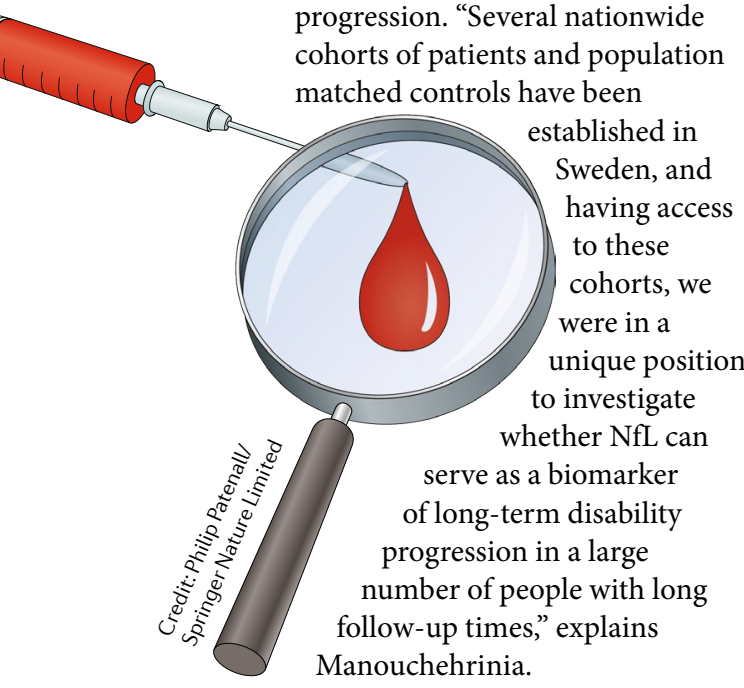

The study included 4,385 patients with MS and 1,026 healthy controls who were matched for age and sex. The researchers measured NfL levels in plasma samples obtained from patients at the time of their MS diagnosis, or at the equivalent age in controls. NfL levels were then compared with Expanded Disability Status Scale (EDSS) scores for each participant over a median follow-up of 5 years. Among individuals with MS, those whose plasma levels of $\mathrm{NfL}$ at diagnosis were higher than those of agematched controls were at greater risk of reaching EDSS milestones during follow-up than were individuals whose NfL levels were not elevated.

"We found that a simple blood test very early on in the course of MS can predict the future of a patient with MS in the long-term," says Manouchehrinia. "This is not without uncertainty and errors, but can help practicing neurologists and patients with MS make better decisions very early on that hopefully will lead to a milder course of MS."

Now, the team want to learn more about the relationship of NfL to MS disability and also determine whether its blood levels can tell us about cognitive function. "In some patients with MS, the risk of cognitive impairment is much bigger than physical disability," says Manouchehrinia. "We would like to know whether NfL can predict the risk of cognitive decline too."

The second study, led by Alberto Gajofatto, focused on miRNAs as markers of MS clinical course. Altered expression of miRNAs has previously been associated with diseases that involve disruption of the immune system. "Circulating miRNAs are present in several body fluids, including serum, where they are exceptionally stable," says Gajofatto. “This makes them potential candidates for clinical application in MS."

In their exploratory study, Gajofatto and colleagues measured the serum levels of several candidate miRNAs - chosen on the basis of previous evidence - in 74 people with MS, 13 of whom had primary or secondary progressive MS.

Levels of miR-128-3p were higher in people with progressive MS than in people with relapsingremitting MS. Among patients with relapsing-remitting MS, serum levels of miR-128-3p were higher in people who experienced relapses during the follow-up period than in people who experienced no relapses. Furthermore, levels of miR-128-3p correlated inversely with relapse rate.

"Although preliminary, these findings indicate that miR-128-3p is a possible biomarker of relapse risk in MS," says Gajofatto. "Future research could include the identification of miRNAs or panels of miRNAs that could be assayed in easily accessible blood samples to predict relapse risk and other measures of disease severity."

ORIGINAL ARTICLES Zanoni, M. et al. Up regulated serum miR-128-3p in progressive and relapse-free multiple sclerosis patients. Acta Neurol. Scand. https://doi.org/10.1111/ane.13288 (2020)| Manouchehrinia, A. et al. Plasma neurofilament light levels are associated with the risk of disability in multiple sclerosis. Neurology https://doi.org/ 10.1212/WNL.00000000000009571 (2020) RELATED ARTICLE Khalit, M. et al. Neurofilaments as biomarkers in neurological disorders. Nat. Rev. Neurol. 14, 577-589 (2018) 